\title{
MULTIPLICITY OF SOLUTIONS FOR 2p-ORDER ORDINARY DIFFERENTIAL EQUATION WITH PERIODIC NONLINEARITY
}

\section{YUCHENG BU}

School of Mathematical Science

Nanjing Normal University

Nanjing 210023

P. R. China

Danyang Normal School

Zhenjiang College

Zhenjiang 212300

P. R. China

e-mail: ychbu@126.com

\begin{abstract}
In this paper, a class of $2 p$-order ordinary differential equation with periodic nonlinearity are concerned. Several assumptions are made by an index theory for the corresponding linear homogeneous equation. Under these assumptions, a multiplicity result of solutions for the concerned equation is obtained by applying Lyapunov-Schmidt reduction and a careful analysis of the oscillatory behaviour of the bifurcation equation induced by the concerned equation.
\end{abstract}

2010 Mathematics Subject Classification: 34B15, 35B34.

Keywords and phrases: ordinary differential equation, index theory, Lyapunov-Schmidt reduction, resonance, periodic nonlinearity.

This work is supported by Project of Building Scientific Research Team of Zhenjiang College (ZJCKYTD20).

Received March 17, 2017; Revised April 19, 2017

(C) 2017 Scientific Advances Publishers 


\section{Introduction and Main Result}

In this paper, we are concerned with the boundary-value problem

$$
\left\{\begin{array}{l}
(-1)^{p+1} x^{(2 p)}(t)+q(t) x(t)+g(x(t))=h(t), \quad t \in[0,1], \\
x^{(2 k-2)}(0)=0=x^{(2 k-2)}(1), \quad k=1,2, \cdots, p,
\end{array}\right.
$$

where $p$ is a positive integer, $q \in L^{\infty}[0,1], g$ is a continuous and $T$-periodic function with zero mean value, not identically zero, $h \in C[0,1]$.

Clearly, if $g \equiv 0 \equiv h$, then (1.1) will become the linear homogeneous equation

$$
\left\{\begin{array}{l}
(-1)^{p+1} x^{(2 p)}(t)+q(t) x(t)=0, \quad t \in[0,1] \\
x^{(2 k-2)}(0)=0=x^{(2 k-2)}(1), \quad k=1,2, \cdots, p .
\end{array}\right.
$$

Some special cases of (1.1) have been discussed in many papers. Specially, the existence results of solutions for (1.1) where $p=1$ and $q$ is identically equal to a real number (including its generalizations with the form of partial differential equations) are found in [17, 18, 20, 21]. The multiplicity result of solutions for (1.1) where $p=1$ and $q \in C^{1}\left([0,1] ; \mathbf{R}^{+}\right)$was first studied in [19], and the technique from bifurcation theory has been successfully employed in the course of its proof. Later on, (1.1) where $p=1$ and $q \equiv 1$ was discussed in [3], and the proof of its main result combined Lyapunov-Schmidt reduction together with a careful analysis of the oscillatory behaviour of the socalled bifurcation equation induced by (1.1). This method was employed to several kinds of equations (see [5-9]) and the similar results were obtained correspondingly. Meanwhile, another multiplicity result of solutions for the same problem was proved in [4] by studying the restriction of the corresponding functional and using Lagrange multipliers method. In addition, a generalization of the results in [3, 19] where the corresponding nontrivial solution to the linear part changes sign was given in [15] and its proof was used Lyapunov-Schmidt 
reduction and asymptotic methods based on the stationary phase argument. In [16], the so-called dynamical version of the classical Lyapunov-Schmidt procedure was employed and the multiplicity results of solutions for the same problem was also obtained. Multiplicity questions for problems similar to (1.1) where $p=1$ (including partial differential equations) have been also discussed in $[2,10,11]$. In this paper, we consider the multiplicity of solutions for (1.1). To the best of our knowledge, (1.1) has not been previously considered in the literature.

This paper is organized as follows. In Section 2, we first recall a classification theory for linear $2 p$-order Hamiltonian systems which is the higher dimensional case of (1.2) in [14] (especially, the case of $p=1$ is seen in $[12,13])$. Thanks to the classification theory, we make some assumptions of $q$ in (1.1). Then, we quote an important lemma in [1] which can help us find the nontrivial solutions of (1.1). In addition, we concern a decomposition of a closed subspace of the Banach space $C[0,1]$ which depends on the nontrivial solution of (1.2), and rewrite (1.1) in another form. In Section 3, we state our main result and give its proof by using Lyapunov-Schmidt reduction and a careful analysis of the oscillatory behaviour of the bifurcation equation induced by (1.1).

\section{Preliminaries}

First, let us recall several definitions and propositions of an index classification theory for linear $2 p$-order Hamiltonian systems in [14]. For the sake of our need, we state them only in one dimensional case. According to these results we can make some assumptions of $q$ in (1.1).

Definition 2.1 ([14]). For any $A \in L^{\infty}[0,1]$, we define

$$
q_{A}(x, y)=\int_{0}^{1}\left[x^{(p)}(t) \cdot y^{(p)}(t)-A(t) x(t) \cdot y(t)\right] d t, \quad \forall x, y \in E,
$$

where $E \equiv\left\{x \in H^{p}[0,1] \mid x^{(2 j)}(0)=0=x^{(2 j)}(1), 0 \leq j<\frac{p}{2}\right\}$ is a Hilbert space with the norm $\|x\|=\left(\int_{0}^{1}\left(\left|x^{(p)}(t)\right|^{2}+|x(t)|^{2}\right) d t\right)^{\frac{1}{2}}$. For any $x, y \in E$, 
if $q_{A}(x, y)=0$, we say that $x$ and $y q_{A}$-orthogonal. For any $x \in E_{1}$ and $y \in E_{2}$, where $E_{1}$ and $E_{2}$ are the two subspaces of $E$, if $q_{A}(x, y)=0$, we say that $E_{1}$ and $E_{2} q_{A}$-orthogonal.

Proposition 2.2 ([14]). For any $A \in L^{\infty}[0,1], E$ has a $q_{A}$-orthogonal decomposition

$$
E=E^{+}(A) \oplus E^{0}(A) \oplus E^{-}(A),
$$

such that

(i) $q_{A}(x, x)>0, \forall x \in E^{+}(A), x \neq \theta$;

(ii) $q_{A}(x, x)=0, \forall x \in E^{0}(A)$;

(iii) $q_{A}(x, x)<0, \forall x \in E^{-}(A), x \neq \theta$.

Moreover, $E^{0}(A)$ and $E^{-}(A)$ are finitely dimensional.

Definition 2.3 ([14]). For any $A \in L^{\infty}[0,1]$, we define $i_{p}(A)=\operatorname{dim} E^{-}(A)$, $\nu_{p}(A)=\operatorname{dim} E^{0}(A)$. We call $i_{p}(A)$ and $\nu_{p}(A)$ the index and nullity of $A$, respectively.

Proposition 2.4 ([14]). For any $A \in L^{\infty}[0,1], \nu_{p}(A)$ is the dimension of the solution space of $(-1)^{p+1} x^{(2 p)}(t)+A(t) x(t)=0, x^{(2 k-2)}(0)=0=x^{(2 k-2)}(1)$, $k=1,2, \cdots, p$, and $i_{p}(A)=\sum_{\lambda<0} \nu_{p}(A+\lambda)$.

The following proposition is a direct corollary of Proposition 5.1.2, Definition 2.2.1 and Remark 2.2.1 in [13].

Proposition 2.5 ([13]). For any $A \in L^{\infty}[0,1], i_{1}(A)=k, \nu_{1}(A)=1$ if and only if $A \in H_{k} \equiv\left\{A \in L^{\infty}[0,1]\right.$ the nontrivial solution of (1.2) where $p=1$ has exactly $k$ zeros in $(0,1)\}$. 
Proposition 2.6. For some $A \in L^{\infty}[0,1], \nu_{1}(A)=1, A(t)>0$ for any $t \in[0,1]$, and $e(t)$ is a nontrivial solution of (1.2), then $e^{\prime}$ has only finite zeros in $(0,1)$ with $e^{\prime}(t)^{2}-e(t) \cdot e^{\prime \prime}(t)>0$.

Proof. We introduce the Prüfer transformation

$$
\rho(t)=\left(e(t)^{2}+e^{\prime}(t)^{2}\right)^{\frac{1}{2}} \text { and } \phi(t)=\operatorname{arccot} \frac{e^{\prime}(t)}{e(t)},
$$

which is a continuous function and satisfies $\phi(0) \in[0,2 \pi)$ with $e(0)=\rho(0) \sin \phi(0), \dot{e}(0)=\rho(0) \cos \phi(0)$. Then,

$$
\begin{gathered}
e(t)=\rho(t) \sin \phi(t), e^{\prime}(t)=\rho(t) \cos \phi(t), \\
\phi^{\prime}(t)=\cos ^{2} \phi(t)+A(t) \sin ^{2} \phi(t) .
\end{gathered}
$$

We know that $e^{\prime}=0$ if and only if $\phi(t)=\frac{\pi}{2}+m \pi, m \in \mathbf{Z}$ from (2.1). On the other hand, thanks to $A(t)>0$, we find that $\phi(t)$ is a strictly monotone increasing function from (2.2). Moreover, the continuity of $\phi$ implies that $\phi$ is also bounded on $[0,1]$. Hence, $e^{\prime}$ has only finite zeros. In addition, (2.1) implies $e^{\prime}(t)^{2}-e(t) \cdot e^{\prime \prime}(t)>0$.

Thanks to the results of the index classification theory mentioned above, we can make some assumptions of $q$ so as to discuss the multiplicity of solutions for (1.1). The first one is that

(Q1) $i_{p}(q)=0, \nu_{p}(q)=1$, where $q \in L^{\infty}[0,1]$

Remark 2.7. Assumption (Q1) is natural. Moreover, $v_{p}(q)=1$ shows that the solution space of (1.2) is one dimensional via Proposition 2.4, and any nontrivial solution of (1.2) where $p=1$ has no zero in $(0,1)$ via Proposition 2.5. In other words, any nontrivial solution of (1.2) does not change its sign in $(0,1)$. Hence, without loss of generality, we further assume that 
(Q2) there exists a nontrivial solution of (1.2) denoted by $\bar{x}$ such that $\bar{x}(t)>0$ for any $t \in(0,1)$ and $\int_{0}^{1} \bar{x}^{2}(t) d t=1$.

Remark 2.8. We can illustrate the reasonability of Assumptions (Q2) and (Q1) further. For any given $p$, set $q_{1}(t) \equiv \pi^{2 p}$ on $[0,1]$. Then, $i_{p}\left(q_{1}\right)=0, \nu_{p}\left(q_{1}\right)=1$ and $x=\sqrt{2} \sin \pi t$ is a nontrivial solution of (1.2) where $q=q_{1}$. Clearly, $x>0$ in $(0,1)$ at the moment. However, if we set $q_{2}(t) \equiv(2 \pi)^{2 p}$ on $[0,1]$, then $i_{p}\left(q_{2}\right)=1, \nu_{p}\left(q_{2}\right)=1$ and $x=\sqrt{2} \sin 2 \pi t$ is also a nontrivial solution of (1.2) where $q=q_{1}$. Now we find that $x$ changes its sign in $(0,1)$. In addition, we have $\int_{0}^{1} x^{2}(t) d t=1$.

Remark 2.8 shows that for any given $p$, the derivative of the nontrivial solution $x=\sqrt{2} \sin 2 \pi t$ of (1.2) where $q(t) \equiv \pi^{2 p}$ has exactly one zero in $(0,1)$. Moreover, $x$ satisfies $x^{\prime}(t)^{2}-x(t) \cdot x^{\prime \prime}(t)=8 \pi^{2}>0$. Together with this fact, Proposition 2.6 enlightens us to make the following assumption:

(Q3) $q \neq 0$ on $[0,1]$, and $\bar{x}$ has only finite zeros in $(0,1)$. Moreover, $\bar{x}^{\prime}(t)^{2}-\bar{x}(t) \cdot \bar{x}^{\prime \prime}(t)>0$ on $[0,1]$.

In addition, we list on the assumption of $g$ which has been stated at the beginning of this paper.

(G) $g$ is a continuous and $T$-periodic function with zero mean, not identically zero.

Secondly, we need to recall a lemma in [1] (see Lemma 1.2) because it is crucial to our arguments. Meanwhile, some prior statements are prepared. 
$X$ is an arbitrary Banach space. $L: X \rightarrow X$ is a Fredholm operator, the domain, kernel and range of $L$ is denoted by $D(L), N(L)$, and $R(L)$, respectively. Of course, $D(L) \subseteq X$. Then, there exists a closed linear subspace $V$ and a linear subspace $U$ of $X$, respectively, such that $X=N(L) \oplus V$ and $X=U \oplus R(L)$. Let $Q$ be the projection onto $R(L)$, and the restriction of $L$ to $D(L) \cap V$ has a continuous inverse $H: R(L) \rightarrow V$. Moreover, let $F$ be a nonlinear map with domain $D(F)=X$ such that $D(L) \cap D(F) \neq \emptyset$.

Lemma 2.9 ([1]). For $L, V, Q, H, F$ mentioned above, assume that the map $H Q F: X \rightarrow V$ is uniformly bounded and compact on bounded sets. Let

$$
\sum=\{(s, v) \in \mathbf{R} \times V \mid v+H Q F(s u+v)=0\},
$$

where $u \in N(L)$. Then, for any $\alpha>0$, there exists a connected subset $\sum_{\alpha}$ of $\sum$ such that $\operatorname{proj}_{\mathbf{R}} \supseteq[-\alpha, \alpha]$.

Finally, let us consider to decompose a subspace space of $C[0,1]$ and rewrite (1.1) correspondingly.

As we all know, the Banach space $C[0,1]$ possesses the usual norm $\|x\|_{\infty}=\max _{t \in[0,1]}|x(t)|$. Set

$$
X=\{x \in C[0,1] \mid x(0)=0=(1)\} .
$$

Then $X$ is also a Banach space with the same norm of $C[0,1]$. Moreover, set

$$
W=\left\{x \in X \mid \int_{0}^{1} x(t) \cdot \bar{x}(t) d t=0\right\}
$$

which is a closed subspace of $X$. Then, we can decompose $X$ as

$$
X=\operatorname{span}\{\bar{x}(\cdot)\} \oplus W .
$$


Hence, for any $h \in X, h$ has a (unique) decomposition

$$
h(t)=r \bar{x}(t)+\tilde{h}(t), \quad r \in \mathbf{R}, \quad \tilde{h} \in W,
$$

and (1.1) can be rewritten in the form

$$
\left\{\begin{array}{l}
(-1)^{p+1} x^{(2 p)}(t)+q(t) x(t)+g(x(t))=r \bar{x}(t)+\tilde{h}(t), \quad t \in(0,1), \\
x(0)=0=x(1)
\end{array}\right.
$$

where $r \in \mathbf{R}, \tilde{h} \in W$.

In next section, we only investigate the solutions of (2.6).

\section{Main Result and its Proof}

Theorem 3.1. If $q$ and $g$ satisfies (Q1) - (Q3) and (G), respectively, then for any given $p, q, g, \tilde{h}$ and each given natural number $n$, there is a positive constant $\varepsilon_{n}$ (depending on $p, q, g, \tilde{h}$, and $n$ ) such that (2.6) has at least $n$ solutions if $0<|r|<\varepsilon_{n}$. Finally, if $r=0$, then (2.6) has infinitely many solutions.

Proof. We will take three steps to complete the proof of Theorem 3.1.

Step 1. For any given $p, q, g$, and $\tilde{h}$, there is the equivalent condition of the existence of solutions for (2.6).

Set $Y=\left\{x \in C^{(2 p-2)}[0,1] \mid x^{(2 k-2)}(0)=0=x^{(2 k-2)}(1), k=1,2, \cdots, p\right\}$, with the norm $\|x\|_{1}=\max _{0 \leq k \leq 2 p-2}\left\|x^{(k)}(t)\right\|_{\infty}$ for any $x \in Y$ and $Z=C^{2 p}[0,1] \cap Y$.

Define the two operators

$L: Z \rightarrow X,(L x)(t)=(-1)^{p+2} x^{(2 p)}(t)-q(t) x(t)$, for all $x \in Z, t \in[0,1]$, 
and

$$
F: Y \rightarrow X,(F x)(t)=r \bar{x}(t)+\tilde{h}(t)-g(x(t)), \text { for all } x \in Y, t \in[0,1]
$$

Clearly, (2.6) is equivalent to the operator equation

$$
L x+F x=0 .
$$

It is well-known that $L$ is a Fredholm operator of index zero from (Q3). Moreover, (Q1) implies $N(L)=\operatorname{span}\{\bar{x}(t)\}$ and Fredholm alternative theorem implies $\operatorname{Im} L=W$. Hence, there exist a continuous projection $P: X \rightarrow X$ such that

$$
R(P)=R(L), R(I-P)=N(L) .
$$

and (3.3) is equivalent to the alternative system

$$
\begin{gathered}
\tilde{x}+\operatorname{KPF}(c \bar{x}+\tilde{x})=0 \quad \text { (auxiliary equation), } \\
(I-P) F(c \bar{x}+\tilde{x})=0 \quad \text { (bifurcation equation), }
\end{gathered}
$$

where $K$ is the inverse of $L: Z \cap W \rightarrow R(L)$, and any $x \in X$ is written in the form $x(t)=c \bar{x}(t)+\widetilde{x}(t), c \in \mathbf{R}, \widetilde{x}(t) \in W$. (3.2) implies that

$$
K P F(c \bar{x}+\tilde{x})=K P(\tilde{h}+r \bar{x}-g(c \bar{x}+\tilde{x})) .
$$

Then, for any given $c \in \mathbf{R}$, there exists one solution $\tilde{x} \in W$ of (3.5) by applying the Schauder fixed point theorem. We denote by $\sum$ the solution set of (3.5), i.e.,

$$
\sum=\{(c, \tilde{x}) \in \mathbf{R} \times W \mid \tilde{x}=K P F(c \bar{x}+\tilde{x})\}
$$

Thanks to (3.4), we can choose

$$
(I-P) x(t)=\left(\int_{0}^{1} x(t) \cdot \bar{x}(t) d t\right) \bar{x}(t), \text { for all } x \in X .
$$


Then, we have

$$
r=\int_{0}^{1} g(c \bar{x}(t)+\widetilde{x}(t)) \bar{x}(t) d t
$$

from (3.6) and (Q2). Hence, we obtain an equivalent condition of the existence of solutions for (2.6):

For any given $p, q, g$, and $\tilde{h},(2.6)$ has one solution if and only if $r$ belongs to the range of the function $\Gamma: \sum \rightarrow \mathbf{R}$, defined by

$$
\Gamma(c, \tilde{x})=\int_{0}^{1} g(c \bar{x}(t)+\tilde{x}(t)) \bar{x}(t) d t
$$

Moreover, $\Gamma\left(\sum\right)$ is a bounded interval. Indeed, let $r_{1}, r_{2} \in \Gamma\left(\sum\right)$ and $r_{1} \leq r_{2}$. Then, for $h_{1}=r_{1} \bar{x}(t)+\tilde{h}(t), h_{2}=r_{2} \bar{x}(t)+\tilde{h}(t)$, (3.7) shows that (2.6) has at least one solution $\hat{x}(t)$ and $\check{x}(t)$, respectively. Hence, for any $h=r \bar{x}(t)+\tilde{h}(t), r_{1} \leq r \leq r_{2}$, (2.6) has a lower solution $\hat{x}(t)$ and an upper solution $\check{x}(t)$ from (Q2). That is, (2.6) has at least one solution. Therefore, $r \in \Gamma\left(\sum\right)$. The arbitrariness of $r$ deduce that $\Gamma\left(\sum\right)$ is an interval. In addition, $(\mathrm{G})$ implies that $\Gamma\left(\sum\right)$ is also bounded.

Step 2. (2.6) has at least $n$ solutions of if $|r| \leq \varepsilon_{n}$, where $\varepsilon_{n}$ will be given later on.

Let us define the following two functions:

$$
p_{1}: \sum \rightarrow \mathbf{R}, \quad \text { by } p_{1}(c, \tilde{x})=c, \quad \text { for all }(c, \tilde{x}) \in \sum
$$

and

$$
p_{2}: \sum \rightarrow \operatorname{ker} P, \quad \text { by } p_{2}(c, \tilde{x})=\tilde{x}, \quad \text { for all }(c, \tilde{x}) \in \sum \text {. }
$$


Combining with (G), (3.5) implies that there is a constant $M_{0}>0$, independent of $c \in \mathbf{R}$, such that

$$
\|\widetilde{x}\|_{\infty} \leq M_{0},\left\|\tilde{x}^{\prime}\right\|_{\infty} \leq M_{0},\left\|\tilde{x}^{\prime \prime}\right\|_{\infty} \leq M_{0}, \quad \text { for all } \tilde{x} \in p_{2}\left(\sum\right) .
$$

It is easy to see that there exists a constant $c_{0}^{-}>0$ (depending on $p$ and $M_{0}$ ) such that for any $c \geq c_{0}^{-}$,

$$
\left\|\frac{c \bar{x}^{\prime}(t)+\tilde{x}^{\prime}(t)}{\|c \bar{x}+\widetilde{x}\|_{\infty}}-\frac{\bar{x}^{\prime}(t)}{\|\bar{x}\|_{\infty}}\right\|_{\infty}
$$

is small. In addition, we know that $\bar{x}^{\prime}$ has only finite zeros in $(0,1)$ from (Q3). Hence, any zero $t_{1}$ of $c \bar{x}^{\prime}(t)+\tilde{x}^{\prime}(t)$ must be close to only one zero $t_{0}$ of $\bar{x}^{\prime}(t)$ for any $c \geq c_{0}^{-}$and any $\tilde{x} \in p_{2}\left(\sum\right)$. In other words, $c \bar{x}^{\prime}(t)+\tilde{x}^{\prime}(t)$ has only finite zeros in $(0,1)$ for any $c \geq c_{0}^{-}$and any $\tilde{x} \in p_{2}\left(\sum\right)$. Therefore, let $G$ be a primitive of $g$, if $(c, \tilde{x}) \in \sum$ and $c \geq c_{0}^{-}$, then

$$
\begin{aligned}
\Gamma(c, \tilde{x})= & \int_{0}^{1}\left[-G(c \bar{x}(t)+\tilde{x}(t))+G\left(\|c \bar{x}+\tilde{x}\|_{\infty}\right)\right] \\
& \times \frac{c\left[\bar{x}^{\prime}(t)^{2}-\bar{x}(t) \cdot \bar{x}^{\prime \prime}(t)\right]+\bar{x}^{\prime}(t) \cdot \tilde{x}^{\prime}(t)-\bar{x}(t) \cdot \tilde{x}^{\prime \prime}(t)}{\left(c \bar{x}^{\prime}(t)+\tilde{x}^{\prime}(t)\right)^{2}} d t .
\end{aligned}
$$

Set

$$
M_{1}=\max \left\{1,\|\bar{x}\|_{\infty},\left\|\bar{x}^{\prime}\right\|_{\infty}\right\} \text { and } M_{2}=\min _{[0,1]}\left(\bar{x}^{\prime}(t)^{2}-\bar{x}(t) \cdot \bar{x}^{\prime \prime}(t)\right) .
$$

Clearly, $M_{1}>0$. (Q3) implies $M_{2}>0$. Denote $c_{0}^{+}=\frac{2 M_{1} M_{0}+1}{M_{2}}$. (3.9) and (3.11) imply that

$$
c\left[\bar{x}^{\prime}(t)^{2}-\bar{x}(t) \cdot \bar{x}^{\prime \prime}(t)\right]+\bar{x}^{\prime}(t) \cdot \widetilde{x}^{\prime}(t)-\bar{x}(t) \cdot \widetilde{x}^{\prime \prime}(t)>0,
$$

for any $c \geq c_{0}^{+}$. 
Now let us set

$$
c_{0}=\max \left\{c_{0}^{-}, c_{0}^{+}\right\}, G\left(s_{1}\right)=\max _{[0, T]} G \text {, and } G\left(s_{2}\right)=\min _{\left[M_{1} T,\left(M_{1}+1\right) T\right]} G \text {. }
$$

Then, there exists some natural number $n_{1}$ such that $s_{1}+n_{1} T \geq c_{0} M_{1}+M_{0}$.

Hence, we can set

$$
s_{1}^{-}=\frac{s_{1}+n_{1} T-M_{0}}{M_{1}}, \quad s_{1}^{+}=\frac{s_{1}+n_{1} T+M_{0}}{M_{1}} .
$$

For the spaces $X, W$ and the operators $L, P, F, K$ mentioned in (2.3), (2.4), (3.1), (3.4), and (3.5), respectively, we can verify that $K P F$ is uniformly bounded and compact on bounded sets. Therefore, we can deduce from Lemma 2.9 that for $L$ there exists a connected subset $\sum_{1}$ of $\sum$ such that $p_{1}\left(\sum_{1}\right) \supseteq\left[-s_{1}^{+}, s_{1}^{+}\right] \supseteq\left[s_{1}^{-}, s_{1}^{+}\right]$and $\left(s_{1}^{-}, \tilde{x}_{s_{1}^{-}}\right),\left(s_{1}^{+}, \tilde{x}_{s_{1}^{+}}\right) \in \sum_{1}$. Since $\left\|s_{1}^{-} \bar{x}+\widetilde{x}_{s_{1}^{-}}\right\|_{\infty} \leq s_{1}+n_{1} T,\left\|s_{1}^{+} \bar{x}+\widetilde{x}_{s_{1}^{+}}\right\|_{\infty} \geq s_{1}+n_{1} T$ and the norm function $\|\cdot\|_{0}$ is continuous, there exists $\left(c_{1}, \tilde{x}_{c_{1}}\right) \in \sum_{1}$ with $\left\|c_{1} \bar{x}(t)+\tilde{x}_{c_{1}}(t)\right\|_{0}=s_{1}+n_{1} T$ such that

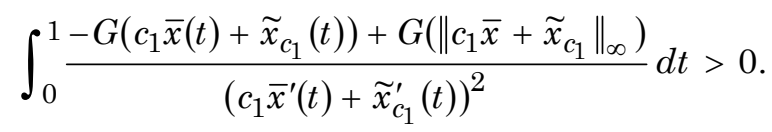

(3.13) combining with (3.10) and (3.12) implies $\Gamma\left(c_{1}, \tilde{x}_{c_{1}}\right)>0$.

Similarly, we also set

$$
s_{2}^{-}=\frac{s_{2}+n_{1} T-M_{0}}{M_{0}}, \quad s_{2}^{+}=\frac{s_{2}+n_{1} T+M_{0}}{M_{1}} .
$$

Then, there exists another connected subset $\sum_{2}$ of $\sum$ such that $p_{1}\left(\sum_{2}\right) \supseteq\left[-s_{2}^{+}, s_{2}^{+}\right] \supseteq\left[s_{2}^{-}, s_{2}^{+}\right]$and $\left(s_{2}^{-}, \tilde{x}_{s_{2}^{-}}\right),\left(s_{2}^{+}, \tilde{x}_{s_{2}^{+}}\right) \in \sum_{2}$. Therefore, there exists $\left(c_{2}, \tilde{x}_{c_{2}}\right) \in \sum_{2}$ with $\left\|c_{2} \bar{x}(t)+\tilde{x}_{2}(t)\right\|_{\infty}=s_{2}+n_{1} T$ such that 


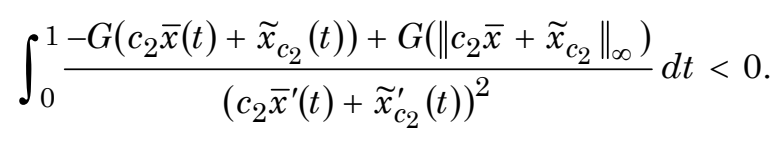

Together with (3.10) and (3.12), (3.14) implies that $\Gamma\left(c_{2}, \tilde{x}_{c_{2}}\right)<0$.

Now for the interval $\left[s_{1}^{-}, s_{2}^{+}\right]$, there exists another connected subset $\sum_{1,2}$ of $\sum$ such that $p_{1}\left(\sum_{1,2}\right) \supseteq\left[s_{1}^{-}, s_{2}^{+}\right]$and $\Gamma\left(\sum_{1,2}\right) \supseteq\left[-\delta_{1}, \delta_{1}\right]$, where

$$
\delta_{1}=\min \left\{\Gamma\left(c_{1}, \tilde{x}_{c_{1}}\right),-\Gamma\left(c_{2}, \tilde{x}_{c_{2}}\right)\right\}
$$

Similarly, we can denote

$$
G\left(s_{2 i-1}\right)=\max _{\left[(i-1)\left(M_{1}+1\right) T,\left((i-1) M_{1}+i\right) T\right]} G, \quad i=2, \cdots, n,
$$

and

$$
G\left(s_{2 i}\right)=\min _{\left[\left(i\left(M_{1}+1\right)-1\right) T, i\left(M_{1}+1\right) T\right]} G, \quad i=2, \cdots, n .
$$

Then, there exists some natural numbers $n_{i}$ such that $s_{2 i-1}+n_{i} T \geq$ $c_{0} M_{1}+M_{0}$. In addition, we can obtain the intervals $\left[s_{2 i-1}^{-}, s_{2 i}^{+}\right]$, where

$$
s_{2 i-1}^{-}=\frac{s_{2 i-1}+n_{i} T-M_{0}}{M_{1}}, \quad s_{2 i}^{+}=\frac{s_{2 i}+n_{i} T+M_{0}}{M_{1}}, \quad i=2, \cdots, n .
$$

For each interval mentioned above, there is a connected subset $\sum_{2 i-1,2 i}$ of $\sum$ such that $p_{1}\left(\sum_{2 i-1,2 i}\right) \supseteq\left[-s_{2 i-1}^{+}, s_{2 i}^{+}\right] \supseteq\left[s_{2 i-1}^{-}, s_{2 i}^{+}\right]$ and $\Gamma\left(\sum_{2 i-1,2 i}\right) \supseteq\left[-\delta_{i}, \delta_{i}\right]$, where

$$
\delta_{i}=\min \left\{\Gamma\left(c_{2 i-1}, \tilde{x}_{c_{2 i-1}}\right),-\Gamma\left(c_{2 i}, \tilde{x}_{c_{2 i}}\right)\right\}, i=2, \cdots, n,
$$

$\left(c_{2 i-1}, \tilde{x}_{c_{2 i-1}}\right) \in \sum_{2 i-1},\left(c_{2 i}, \tilde{x}_{c_{2 i}}\right) \in \sum_{2 i}$, and $\sum_{2 i-1}, \sum_{2 i}$ are the connected subsets of $\sum$. Set $\varepsilon_{n}=\min \left\{\delta_{1}, \delta_{2}, \cdots, \delta_{n}\right\}$. Hence, if $|r|<\varepsilon_{n}$, then (2.6) has at least $n$ solutions $x$ of the form

$$
x=c_{i} \bar{x}+\tilde{x} \quad \text { with } \quad c_{i} \in\left[s_{2 i-1}^{-}, s_{2 i}^{+}\right](i=1,2, \cdots, n) .
$$


Step 3. If $r=0$, then (2.6) has infinitely many solutions.

The structure of $x$ in (3.15) implies that the conclusion mentioned above holds naturally.

\section{References}

[1] H. Amann, A. Ambrosetti and G. Mancini, Elliptic equations with noninvertible Fredholm linear part and bounded nonlinearities, Math. Z. 158 (1978), 179-194.

[2] D. Bonheure, C. Fabry and D. Ruiz, Problem at resonance for equations with periodic nonlinearities, Nonlinear Anal. 55 (2003), 557-581.

[3] A. Cañada and F. Roca, Existence and multiplicity of solutions of some conservative pendulumtype equations with homogeneous Dirichlet conditions, Differential Integral Equation 10 (1997), 1113-1122.

[4] A. Cañada and F. Roca, Qualitative properties of some noncoercive functionals arising from nonlinear boundary value problems, Nonlinear Anal. 37 (1999), 861-873.

[5] A. Cañada, Nonlinear ordinary boundary value problems under a combined effect of periodic and attractive nonlinearities, J. Math. Annal. Appl. 243 (2000), 174-189.

[6] A. Cañada and A. Ureña, Solvability of some pendulum-type equations with linear damping and homogeneous Dirichlet conditions, Nonlinear Anal. 49 (2002), 1149-1165.

[7] A. Cañada and D. Ruiz, Resonant nonlinear boundary value problems with almost periodic nonlinearity, Bull. Belg. Math. Soc. 9 (2002), 193-204.

[8] A. Cañada and D. Ruiz, Asymptotic analysis of oscillating parametric integrals and ordinary boundary value problems at resonance, J. Math. Anal. Appl. 313 (2006), 218-233.

[9] A. Cañada, Multiplicity results near the principal eigenvalue for boundary-value problems with periodic nonlinearity, Math. Nachr. 280(3) (2007), 235-241.

[10] S. Chen and S. Li, Splitting lemma in the infinity and a strong resonant problem with periodic nonlinearlity, Nonlinear Anal. 65 (2006), 567-582.

[11] S. Chen and S. Li, Splitting lemma at infinity and a strong resonant problem with periodic nonlinearlity, Calc. Var. 27(1) (2006), 105-123.

[12] Y. Dong, Index theory, nontrivial solutions and asymptotically linear second-order Hamiltonian systems, J. Differ. Equation 214 (2005), 233-255.

[13] Y. Dong, Index Theory for Hamiltonian systems and multiple soluiton problems, Science Press, Beijing 63 (2015), 9-13.

[14] A. Du, Morse Index Theory and Existence of Solutions of Asymptotically Linear $2 p$-order Hamiltonian Systems, Master, Nanjing Normal University, 2008. 
[15] P. Girg, F. Roca and S. Villegas, Semilinear Sturm-Liouville problem with periodic nonlinearity, Nonlinear Anal. 61 (2005), 1157-1178.

[16] Philip Korman, Global solution curves for boundary value problems, with linear part at resonance, Nonlinear Anal. 71 (2009), 2456-2467.

[17] E. Landesman and A. Lazer, Nonlinear perturbations of linear elliptic boundary value problems at resonance, J. Math. Mech. 19 (1970), 609-623.

[18] D. Lupo and S. Solimini, A note on resonance problem, Proceedings of the Royal Society of Edinburgh 102A (1986), 1-7.

[19] R. Schaaf and K. Schimit, A class of nonlinear Sturm-Liouville problems with infinitely many solutions, Trans. Amer. Math. Soc. 306 (1988), 853-859.

[20] S. Solimini, On the solvability of some elliptic partial differential equations with the linear part at resonance, J. Math. Anal. Appl. 117 (1986), 138-152.

[21] J. Ward, A boundary value with a periodic nonlinearity, Nonlinear Anal. 10(2) (1986), 207-213. 